\title{
pcrEfficiency: a Web tool for PCR amplification efficiency prediction
}

\author{
Izaskun Mallona*, Julia Weiss and Marcos Egea-Cortines
}

\begin{abstract}
Background: Relative calculation of differential gene expression in quantitative PCR reactions requires comparison between amplification experiments that include reference genes and genes under study. Ignoring the differences between their efficiencies may lead to miscalculation of gene expression even with the same starting amount of template. Although there are several tools performing PCR primer design, there is no tool available that predicts PCR efficiency for a given amplicon and primer pair.

Results: We have used a statistical approach based on 90 primer pair combinations amplifying templates from bacteria, yeast, plants and humans, ranging in size between 74 and 907 bp to identify the parameters that affect PCR efficiency. We developed a generalized additive model fitting the data and constructed an open source Web interface that allows the obtention of oligonucleotides optimized for PCR with predicted amplification efficiencies starting from a given sequence.

Conclusions: pcrEfficiency provides an easy-to-use web interface allowing the prediction of PCR efficiencies prior to web lab experiments thus easing quantitative real-time PCR set-up. A web-based service as well the source code are provided freely at http://srvgen.upct.es/efficiency.html under the GPL v2 license.
\end{abstract}

\section{Background}

Since the development of quantitative PCR (Q-PCR) in the early nineties [1], it has become an increasingly important method for gene expression quantification. Its aim is to amplify a specific DNA sequence under monitoring and measuring conditions that allow stepwise quantification of product accumulation. Product quantification has fostered the development of analysis techniques and tools. These data mining strategies focus on the cycle in which fluorescence reaches a defined threshold (value called quantification cycle or Cq) [2,3]; with the $C q$ parameter, quantification could be addressed following two approaches: (i) the standard curve method [4] and (ii) the $\Delta \Delta C q$ method [5].

It is worth noting that these classical quantification methods assume that amplification efficiency is constant or even equal to $100 \%$. An efficiency value of $100 \%$ implies that during the exponential phase of the Q-PCR reaction, two copies are generated from every available template. But it has been shown that these assumptions

\footnotetext{
* Correspondence: izaskun.mallona@upct.es

Genetics, Institute of Plant Biotechnology (IBV), Technical University of

Cartagena (UPCT), Campus Muralla del Mar, 30202 Cartagena, Spain
}

are not supported by experimental evidences [6]. With the aim of estimating PCR efficiency, and thus to include it in further analysis procedures, two strategies have been developed: (i) kinetics-based calculation and (ii) standard curve assessment.

Taking into account the reaction kinetics, which is basically equivalent to the bacterial growth formulae [7], amplification efficiency could be visualized in a halflogarithmic plot in which log transformed fluorescence values are plotted against the time (cycle number). In these type of graphic representations, the phase of exponential amplification is linear and the slope of this line is the reaction efficiency [8]. Empirical determinations of amplification efficiencies show that ranges lay between 1.65 and 1.90 (65\% and 90\%) [9]. Standard curve-based calculation method relies on repeating the PCR reaction with known amounts of template. $C q$ values versus template (i.e. reverse transcribed total RNA) concentration input are plotted to calculate the slope. Laboratories where few genes are analyzed for diagnostic may develop standard curves but they are in most cases out of scope for research projects where
C Biomed Central 
tens-hundreds of genes will be tested for changes in gene expression.

Several aspects influence PCR yield and specificity: reagents concentration, primer and amplicon length, template and primer secondary structure, or $\mathrm{G}+\mathrm{C}$ content [10]. The goals of a PCR assay design are: (i) obtaining the desired product without mispriming and (ii) rising yield towards optimum. In most cases, the sequence to amplify is a fixed entity, so setting up an efficient reaction involves changes in reagents concentrations (salts, primers, enzyme) and specifically an optimal primer design. Thus a plethora of primer designing tools have been published, regarding as little as $\mathrm{G}+\mathrm{C}$ content for $T_{m}$ calculation [11,12], evaluating salt composition [13] or even employing Nearest Neighbor modules, which consider primer and salt concentrations [14].

Efficiency values are essential elements in the $\Delta \Delta C q$ method and its variants: relative quantities are calculated using the efficiency value as the base in an exponential equation in which the exponent depends on the $C q$. Thus efficiency strongly influences the relative quantities calculation, which are required to estimate gene expression ratios [5].

In this work, we analysed Q-PCR efficiency values from roughly 4,000 single PCR runs with the aim of elucidating the major variables involved in PCR efficiency. With this data we developed a generalized additive model (GAM), which relies on nonlinear regression analysis, and implemented it in a open, free online web tool allowing efficiency prediction.

\section{Results}

\section{Data overview}

Our data were generated from 90 different amplification products that included four Escherichia coli strains, three Agrobacterium tumefaciens strains [15], three tomato varieties [16], three Petunia hybrida lines [17], one Antirrhinum majus line [18], one Opuntia ficusindica genotype [19] and human liquid cytology samples used to test for Prostate Serum Antigen presence. Efficiencies ranged between 1 (no amplification) and 2 (perfect exponential duplication). We wrote an R script (see Materials and Methods) to extract the inputs for further statistical analysis. The script regarded complete amplicon length, primer sequence, $\mathrm{G}+\mathrm{C}$ content of amplicon and primers, presence of repetitions in the amplicon (N6 or above), primer melting temperature and the 3' terminal, last two nucleotides of each primer and primers tendency to hybridize (Figure 1). Metadata for each PCR reaction included sample origin (i.e. genomic or CDNA), operator involved, species and line or variety, exact sequence amplified, primer length and PCR efficiency; a summary of the data is shown as Additional File 1.

Efficiency dataset had highly repetitive data (presence of ties). As some of our comparisons intended to assess relationships between two quantitative variables, we applied Spearman tests. However, analysis of quantitative versus qualitative data was performed employing Kruskal-Wallis tests. Due to the presence of ties, which hampers the application of rank-based tests, asymptotic tests were applied. p-values were approximated via its

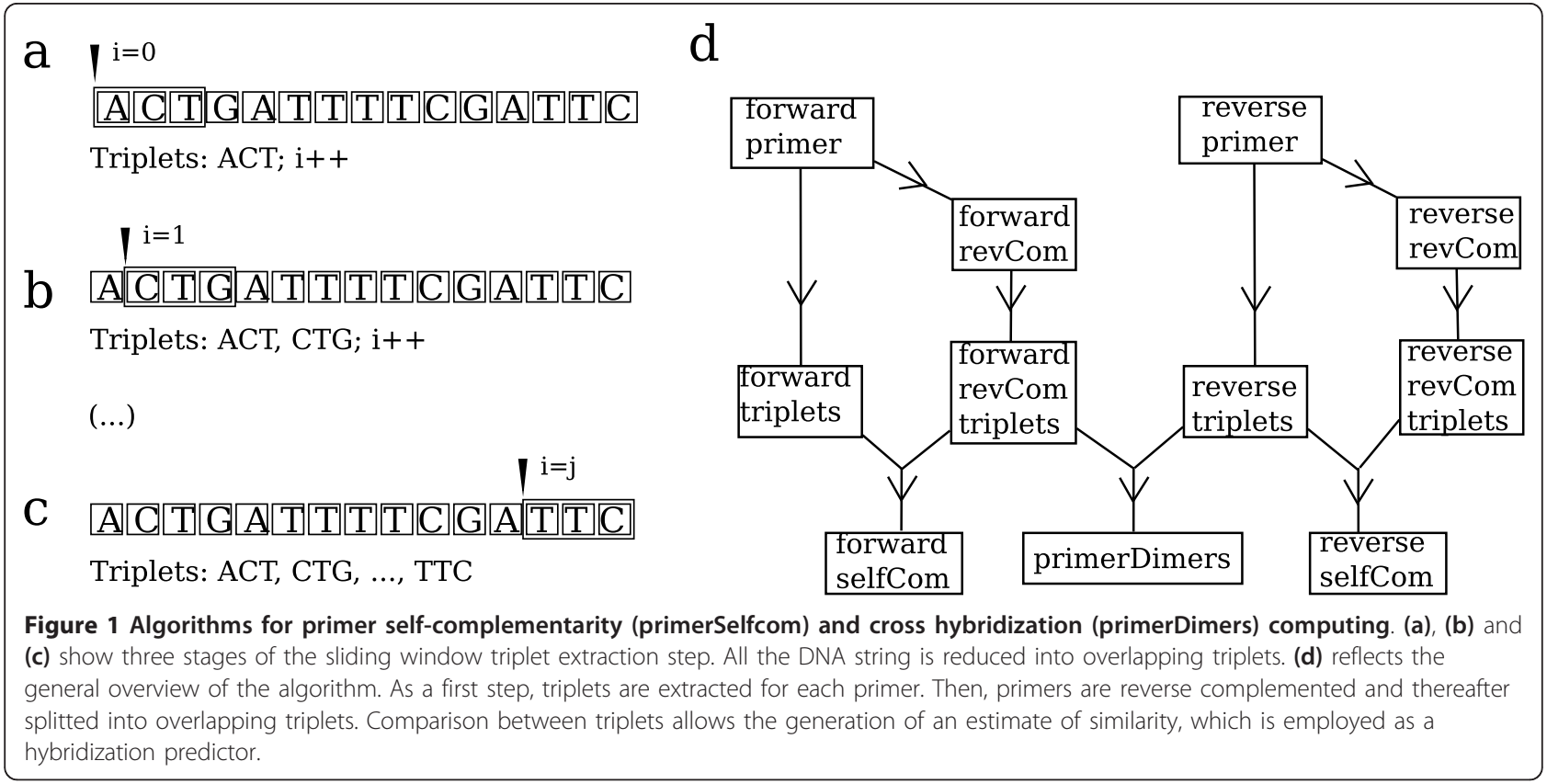


asymptotic distribution and ties were adjusted via random rank averaging. Both procedures are implemented in the coin $\mathrm{R}$ package [20]. We chose a value of 0.05 as cut-off of statistical signification. A summary is shown in Table 1. Post-hoc asymptotic Wilcoxon Mann-Whitney rank sum test were performed to discriminate the contribution of some of the categorical variables, as well to analyzed effect sizes; the statistical outputs are shown as Additional File 2.

In order to build up a predictive statistical model we used a generalized additive modelling procedure as an effective technique for conducting nonlinear regression analysis in which factors were modeled using nonparametric smooth functions. GAM function was implemented by the $\mathrm{R}$ project mgcv package [21], according to the formulation described in [22]. Data fitting is shown in Table 2. Figure 2 shows perspective plot views of the GAM; predicted efficiency is plotted as a response surface defined by the values of two interacting variables.

\section{Statistical modelling}

Model selection was performed according to the Akaike's Information Criterion, a penalized log-likelihood system addressing model goodness and denying low parsimony [23]. We selected a GAM based on the interaction of the length and $\mathrm{G}+\mathrm{C}$ content of the sequence as well, independently, of the primers; and the interaction of the $\mathrm{G}+\mathrm{C}$ imbalance between primers with an estimation of the tendency to produce primer dimers. The R squared parameter of the model is 0.41 , whereas the deviance explained is $42.1 \%$. As the model intends to estimate the PCR efficiency of a set composed by a given amplicon and a given set of oligo primer pairs, it could be validated in terms of ranking performance. We defined a threshold of experimental efficiency measured during the Q-PCR obtaining a decision criterion of adequate PCR performance. We took results below 1.65 (i. e. $65 \%$ ) of efficiency as fails, thus this threshold acts as a binary classifier of success. Receiver Operator Characteristic (ROC) curves are commonly used to analyze how the number of correctly classified positive cases whose predicted efficiency is over the threshold change with the number of incorrectly classified negative examples whose predicted efficiency is below that threshold [24]. This representation is complemented with the precision and recall (PR) curves, which evaluate the relationship between precision (the ability of presenting only relevant items) and recall (true positive rate) [24]. ROC and PR curves are shown in Figure 3; ROC rises rapidly to the the upper-left-hand corner thus reflecting that the falsepositive and false-negative rates are low, whereas the PR curve locates at the upper-right-hand corner and thereafter indicates that most of the items classified as positive are true positives.

Table 1 Statistical results for univariate analysis

\begin{tabular}{|c|c|c|c|c|c|c|}
\hline \multicolumn{7}{|c|}{ Univariate analysis } \\
\hline Variable & value & df & $\rho$ & d & Log Odds & p-value \\
\hline Primers length & $Z=-7.4398$ & - & -0.118 & -0.239 & -0.433 & $1.008 \mathrm{e}-13^{*}$ \\
\hline Sequence length & $Z=-5.423$ & - & -0.086 & -0.173 & -0.314 & $5.86 \mathrm{e}-08^{*}$ \\
\hline Sequence $\mathrm{G}+\mathrm{C}$ content & $Z=-10.2664$ & - & -0.163 & -0.331 & -0.601 & $<2.2 \mathrm{e}-16^{*}$ \\
\hline A repeats & $Z=2.1004$ & - & 0.033 & 0.067 & 0.121 & $0.03569^{*}$ \\
\hline T repeats & $Z=3.9818$ & - & 0.063 & 0.127 & 0.230 & $6.84 \mathrm{e}-05^{*}$ \\
\hline $\mathrm{C}$ repeats & $Z=-5.294$ & - & -0.084 & -0.169 & -0.307 & $1.196 \mathrm{e}-07^{*}$ \\
\hline $\mathrm{G}$ repeats & $Z=-7.1808$ & - & -0.114 & -0.230 & -0.418 & $6.929 e-13^{*}$ \\
\hline Primers $T_{m}$ & $Z=1.4653$ & - & 0.023 & 0.047 & 0.085 & 0.1428 \\
\hline Primers self complementarity & $Z=11.9002$ & - & 0.190 & 0.386 & 0.700 & $<2.2 \mathrm{e}-16^{*}$ \\
\hline Primer dimers & $Z=4.4161$ & - & 0.070 & 0.141 & 0.256 & $1.005 e-05^{*}$ \\
\hline Primer GC imbalance & $Z=11.1367$ & - & 0.177 & 0.360 & 0.654 & $<2.2 \mathrm{e}-16^{*}$ \\
\hline Primers GC content & $Z=4.5921$ & - & 0.073 & 0.147 & 0.266 & $4.388 \mathrm{e}-06^{*}$ \\
\hline Sequence palindromes & $Z=-3.4951$ & - & -0.056 & -0.111 & -0.202 & $0.0004738^{*}$ \\
\hline Species & $\chi^{2}=585.616$ & 9 & - & - & - & $<2.2 \mathrm{e}-16^{*}$ \\
\hline Template & $\chi^{2}=1241.562$ & 12 & - & - & - & $<2.2 \mathrm{e}-16^{*}$ \\
\hline Variety or line & $\chi^{2}=585.8386$ & 18 & - & - & - & $<2.2 \mathrm{e}-16^{*}$ \\
\hline Template source & $\chi^{2}=940.8915$ & 24 & - & - & - & $<2.2 \mathrm{e}-16^{*}$ \\
\hline Operator & $\chi^{2}=727.4887$ & 8 & - & - & - & $<2.2 \mathrm{e}-16^{*}$ \\
\hline Primer's 3 ' last two nucleotides & $\chi^{2}=237.911$ & 15 & - & - & - & $<2.2 \mathrm{e}-16^{*}$ \\
\hline
\end{tabular}

In the case of asymptotic Spearman tests, $Z$ value are shown; $\chi^{2}$ value is written for asymptotic Kruskal-Wallis tests. An asterisk over the p-value reflects a significant influence (p-value $\leq 0.05$ ). Sperman's correlation analysis shows the $\rho$ statistic as well the Cohen's $d$ and Log Odds estimates of effect size. For post-hoc asymptotic Wilcoxon Mann-Whitney rank sum tests and its effect size estimators see Additional File 2. 
Table 2 GAM overview

\begin{tabular}{|c|c|c|c|c|}
\hline \multicolumn{5}{|c|}{ GAM analysis } \\
\hline & Estimate & Std. Error & $\mathrm{t}$ value & $\operatorname{Pr}(>|t|)$ \\
\hline (Intercept) & 1.73825 & 0.00153 & 1136 & $<2 \mathrm{e}-16$ \\
\hline \multicolumn{5}{|c|}{ Approximate significance of smooth terms } \\
\hline & edf & Ref.df & $\mathrm{F}$ & $p$-value \\
\hline s(lengthSequence, gcSequence) & 17.58 & 18.08 & 2.332 & 0.00114 \\
\hline s(primersLength, gcPrimers) & 27.96 & 28.46 & 22.950 & $<2 \mathrm{e}-16$ \\
\hline s(gclmbalance, primerDimers) & 28.83 & 29.33 & 16.717 & $<2 \mathrm{e}-16$ \\
\hline \multicolumn{5}{|c|}{$\begin{array}{c}\text { R-sq. }(\text { adj })=0.41 \text { Deviance explained }=42.1 \% \\
\text { GCV score }=0.0094091 \text { Scale est. }=0.0092293 \mathrm{n}=3944\end{array}$} \\
\hline
\end{tabular}

GAM summary as estimated by the gam function of the mgcv R package. Model formula corresponds to: efficiency s(lengthSequence, gcSequence) $+s$ (primersLength, gcPrimers) $+s$ (gclmbalance, primerDimers).

\section{Implementation}

In order to ease PCR efficiency prediction prior to wetlab PCR set-up, we developed a user-friendly, freely available web tool for assessing primer suitability before and during primer design. For this purpose, we wrote a set of Python/Biopython scripts [25] requested through a Common Gateway Interface (CGI). These scripts were developed to call to the Primer3 software [26], working inside the EMBOSS package [27]. The web tool called PCR efficiency calculator allows primer design starting from a DNA fragment producing a set of theoretical PCR efficiency values. It also predicts PCR efficiency values for preexisting primers and DNA template combinations.

\section{Discussion}

Several tools have been developed to assess primer design procedures. Most of them consider hairpin structure formation avoidance, selection of nucleotides in 3' termini, primer melting temperature, etc. [28]. However, intrinsic amplicon characteristics are not contemplated in primer design. The work we present includes this important parameter as amplification was found to be highly dependent on template structure (Tables 1 and 2 ). Indeed PCR specificity or PCR failure have been found to be dependent on sequence similarity between primers and template, lack of mismatches, or number of priming sites $[29,30]$. Using logistic regression analysis, Benita et al. found that PCR success is highly dependent on regionalized $\mathrm{G}+\mathrm{C}$ content in the template thus showing the importance of template structure as a second step in PCR optimization. Generally, PCR success is evaluated as a dichotomy by presence or absence of product. However in Q-PCR experiments amplification efficiency becomes an important parameter to perform proper statistical analysis that should yield the actual differences in expression between several transcripts. Thus PCR efficiency becomes as important as $C q$ values to determine differential gene expression. Our model showed that $\mathrm{G}+\mathrm{C}$ content in the amplicon plays a key role in PCR efficiency confirming previous work and including it inside as a predictor of PCR efficiency.

Multiple parameters, such sequence palindrome abundance or the nucleotide at the 3' primer termini, were found to significantly contribute to the PCR efficiency when analyzed separately. However, they were not

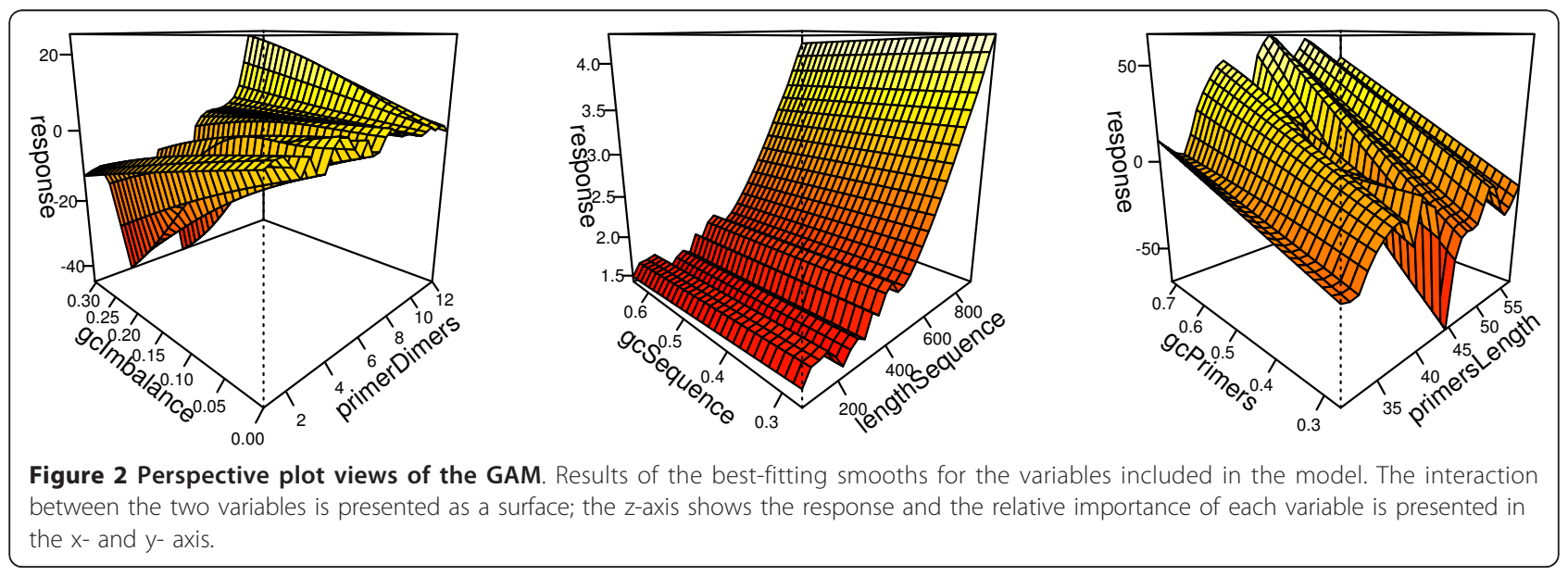




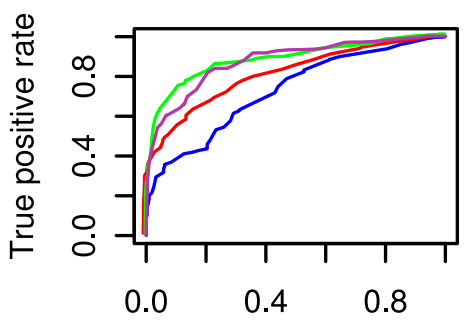

False positive rate

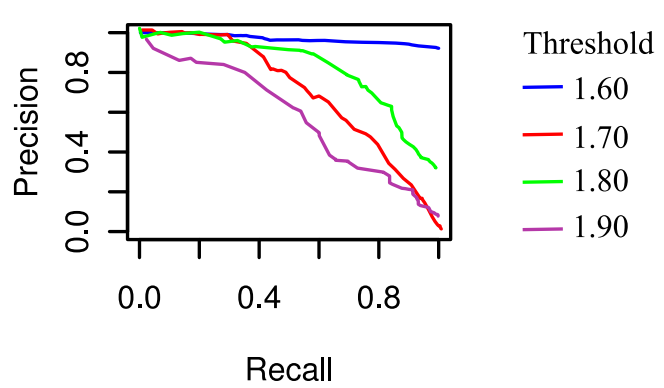

Recall

Figure 3 ROC and PR curves. ROC and PR curves are plotted for various experimental efficiency thresholds, which define the decision criteria of succesful PCR performance. A good behaviour in ROC space is to be in the upper-left-hand corner, whereas in PR space the goal is to locate at the upper-right-hand corner.

significant when included to a multiple component GAM. Unexpectedly, variables such the species or the line the template was extracted from, or the operator involved in the PCR set-up, were found to be significant. Nonetheless, the modelling procedure disregarded these variables, as GAM with and without them did not differ significantly. Thus we ascribe this result as covariation, because each primer pair-amplicon combination is normally used in a certain project which is limited to a species, operator and one or a few lines.

The model presented in this work estimates an efficiency value per PCR reaction regarding three parameters, each of one represents the interaction between two independent variables: the interaction between $\mathrm{G}+\mathrm{C}$ content of the amplicon with its length; the interaction between $\mathrm{G}+\mathrm{C}$ content of the primers with their length; and the interaction between $\mathrm{G}+\mathrm{C}$ content imbalance between primers (gcImbalance; their difference in $\mathrm{G}+\mathrm{C}$ ) with their tendency to hybridize and thus to form primer dimers (primerDimers). Our model gives a high influence to the difference of $\mathrm{G}+\mathrm{C}$ content between primers. Previous works noted that PCR using unequal primer concentrations have better efficiencies when their melting temperatures differ in $\geq 5^{\circ} \mathrm{C}$ [31]. However, when our tool is piped to the Primer3 primer-design work flow, this difference is restricted by the Primer3 algorithms, thus avoiding design of highly unequal primers. Very high or very low amplicon $\mathrm{G}+\mathrm{C}$ content affects amplification success [32,33]. Specially, regionalized $\mathrm{G}+\mathrm{C}$-content has been shown to be relevant in PCR success prediction [34].

The comparison of the model performance in the ROC space discriminates 1.60 as the classifier threshold which leads to the worst model behaviour, but shows only minor differences for the other cut-offs. The analysis of PR curves allows further comparisons and highlights that 1.80 shows the highest degree of resolution. Tuomi and coworkers described 1.80 as boundary for optimized PCR reactions [35].
It is worth noting that the tool developed aids in primer design prior to the wet lab experiments. Since it remains clear that there are physical constraints which establish the maximum PCR efficiency of a given set of one amplicon and a pair of oligos, bias is introduced in many ways (pipetting, reactives, PCR machine, etc.). We would like to point out that our work does not intend to substitute the experimental efficiency calculation nor modify the quantification settings; its aim is to complement the existing primer design tools and thus minimize the need for primer combination testing.

\section{Conclusions}

Using a wide range of amplicons and PCR set-ups, we statistically modelized the response of the PCR efficiency value, a parameter affecting PCR success and involved in effective gene expression quantitation. In order to ease PCR primer design for Q-PCR experiments, the efficiency-predicting model was included in the Primer3 design pipeline and freely provided as a web tool. This tool should help to generate primer combinations with similar theoretical efficiencies to well established PCR primers or to ease multiplex PCR reactions where efficiencies should be similar among templates.

\section{Methods \\ DNA templates}

We used a variety of DNA templates to obtain data for efficiencies including genomic DNA from bacteria, yeasts, plants and humans, and plasmid DNA. Samples using cDNA as template were produced from isolated mRNA from different sources. Synthesis of first strand cDNA was performed from DNAase treated mRNA, using the Maxima kit from Fermentas as described in the protocol. Samples amplified by whole genome amplification using the $\varphi 29$ DNA polymerase were performed with the Genomiphi kit (GE-Healthcare) according to manufacturers manual. A summary of the data is available as Additional File 1. 


\section{Real time PCR}

PCR reactions used were carried out with the SYBR Premix Ex Taq (TaKaRa Biotechnology, Dalian, Jiangsu, China) in a Rotor-Gene 2000 thermocycler (Corbett Research, Sydney, Australia) and analysed with Rotor-Gene analysis software v.6.0 as described before [17]. A second set of reactions was performed with a Mx3000P machine (Stratagene, Amsterdam) and analyzed with the qpcR R package [36]. Reaction profiles used were 40 cycles at $95^{\circ} \mathrm{C}$ for $30 \mathrm{~s}$, an amplicon-specific annealing temperature for $20 \mathrm{~s}$ and amplification at $72^{\circ} \mathrm{C}$. In order to ensure the specificity of the reaction, uniqueness of Q-PCR products were checked by melting analysis (data not shown). Fluorescence data acquisitions during the cycling steps were collected at $72^{\circ} \mathrm{C}$ step, temperature at which eventual primer-dimers should be melted, thus avoiding artifactual contribution to the fluorescence measure. Once finished, analysis was followed by a melting curve whose ramp was delimited between the annealing temperature and $95^{\circ} \mathrm{C}$. Reaction volume was $15 \mu \mathrm{L}$ and each primer was $240 \mathrm{nM}$.

Reaction efficiency was calculated using the amplification curve fluorescence, analyzing each PCR reaction (tube) separately as before [37]. Efficiency value $(E)$ was defined as $E=\frac{F_{n}}{F_{n}-1}$, in which $n$ is determined as the $20 \%$ value of the fluorescence at the maximum of the second derivative curve. Efficiency calculations were performed with the qpcR R package [36]. Curves were formed by 40 points, each one representing a fluorescence measure in each amplification cycle. The RotorGene 2000-based runs were baseline corrected either by standard normalization (substraction of the fluorescence present in the first five cycles of each sample) or by "dynamic tube" normalization (which uses the second derivative of each sample trace to determine the takeoff, thus asigning a threshold separately to each reaction), whereas the Mx3000P were by "adaptive baseline" correction (which assigns a threshold independently to each sample).

\section{Data mining}

Data mining was performed with the $\mathrm{R}$ statistical environment v2.7.1 and v2.10.1 [38] with the following libraries: coin v1.0-4 [20], mgcv v1.7-5 [21], ROCR [39] v1.0-4, compute.es v0.2 [40] and verification v1.31 [41]. The final model was implemented in a CGI server-side set of Python/Biopython scripts interacting with the web browser requests. Source code of both modelling procedures and the server-side application are available at the website.

\section{Additional material}

Additional file 1: Data overview. Data comprise 90 different amplification products that included different template sources. Efficiencies ranged between 1 (no amplification) and 2 (perfect exponential duplication). Measured parameters contained: complete amplicon length (lengthSequence), primer length (forLength and revLength, for the forward and reverse primers respectively), $\mathrm{G}+\mathrm{C}$ content of amplicon and primers (gcSequence and gcPrimers), logical variables showing the presence of $\mathrm{N} 6$ or above repeats (aRepeats, tRepeats, cRepeats, gRepeats); and aCount, tCount, cCount, gCount integer variables regarding the length of the longest repeat found), primer melting temperature (tmForward, tmReverse), tendency to form primer dimers (primerDimers), tendency to selfcomplementarity (primersSelfcom), and the $3^{\prime}$ terminal two nucleotides of each primer (trap3For, trap3Rev) as well the terminal last nucleotides of each primer (trap3LastFor, trap3LastRev). Metadata for each $\mathrm{PCR}$ reaction included the thermocycler used (machine), sample origin (i.e. genomic or CDNA; template) and organ involved (source), person involved (operator), species (species) and line or variety (var), presence of palindromes at the amplicon

(sequencePalindromes), primer length (primersLength), and PCR efficiency (efficiency).

Additional file 2: Post-hoc categorical variable analysis. The variables regarding PCR template (GD, genomic; $C D, C D N A$; plasmid, Escherichia coli plasmid; $y G D$, yeast genomic) and $3^{\prime}$ primer termini ( $U$, purine; $Y$, pyrimidine) were analyzed by asymptotic Wilcoxon Mann-Whitney rank sum tests ( $Z, Z$ value; $p, p$-value), and the effect sizes estimated by the two tailed $p$-value (Cohen's $d$, mean difference; Hedge's $g$, unbiased estimate of $d ; r$, correlation coefficient; and $n$, the total sample size, which is twice the effective sample size when the termini of the two oligos are analyzed).

\section{Acknowledgements and Funding}

This work was funded by Fundación Séneca 11895/PI/09 and MICINN BFU2010-15843. IM received a grant from Fundación Séneca. The authors would like to thank Luis Pedro García and SEDIC for aid in conducting the computational-intensive part of this work, as well the web server set-up. Luciana Delgado-Benarroch is acknowledged for web usability suggestions and Marta Pawluczyk for comments on the manuscript.

\section{Authors' contributions}

IM, ME and JW carried out the design of the study. IM and JW performed the Q-PCR experiments and participated in data collection. IM designed the algorithms, developed the model and programmed the application. IM and ME wrote the manuscript. All authors read and approved the final manuscript.

Received: 12 May 2011 Accepted: 20 October 2011

Published: 20 October 2011

\section{References}

1. Higuchi R, Fockler C, Dollinger G, Watson R: Kinetic PCR analysis: real-time monitoring of DNA amplification reactions. Nature Biotechnology 1993, 11(9):1026-1030.

2. Luu-The V, Paquet N, Calvo E, Cumps J: Improved real-time RT-PCR method for high-throughput measurements using second derivative calculation and double correction. Biotechniques 2005, 38(2):287-293.

3. Bustin S, Benes V, Garson J, Hellemans J, Huggett J, Kubista M, Mueller R, Nolan T, Pfaffl M, Shipley G, et al: The MIQE guidelines: minimum information for publication of quantitative real-time PCR experiments. Clinical Chemistry 2009, 55(4):611.

4. Morrison T, Weis J, Wittwer C: Quantification of low-copy transcripts by continuous SYBR ${ }^{\circledast}$ Green I monitoring during amplification. Biotechniques 1998, 24(6):954-962.

5. Livak K, Schmittgen T: Analysis of relative gene expression data using real-time quantitative PCR and the 2 (-Delta Delta $C(T)$ ) method. Methods 2001, 25(4):402-408. 
6. Ramakers C, Ruijter J, Deprez R, Moorman A: Assumption-free analysis of quantitative real-time polymerase chain reaction (PCR) data. Neuroscience Letters 2003, 339:62-66.

7. Monod J: The growth of bacterial cultures. Annual Reviews in Microbiology $1949,3: 371-394$

8. Schefe J, Lehmann K, Buschmann I, Unger T, Funke-Kaiser H: Quantitative real-time RT-PCR data analysis: current concepts and the novel "gene expression's CT difference" formula. Journal of Molecular Medicine 2006, 84(11):901-910.

9. Tichopad A, Dilger M, Schwarz G, Pfaffl M: Standardized determination of real-time PCR efficiency from a single reaction set-up. Nucleic Acids Research 2003, 31(20):e122.

10. Qu W, Shen Z, Zhao D, Yang Y, Zhang C: MFEprimer: multiple factor evaluation of the specificity of PCR primers. Bioinformatics 2009, 25(2):276.

11. Marmur J, Doty P: Determination of the base composition of deoxyribonucleic acid from its thermal denaturation temperature. Journal of Molecular Biology 1962, 5:109.

12. Wallace R, Shaffer J, Murphy R, Bonner J, Hirose T, Itakura K: Hybridization of synthetic oligodeoxyribonucleotides to DX174 DNA: the effect of single base pair mismatch. Nucleic Acids Research 1979, 6(11):3543.

13. Howley $P$, Israel M, Law M, Martin M: A rapid method for detecting and mapping homology between heterologous DNAs. Evaluation of polyomavirus genomes. Journal of Biological Chemistry 1979, 254(11):4876.

14. Breslauer K, Frank R, Blöcker H, Marky L: Predicting DNA duplex stability from the base sequence. Proceedings of the National Academy of Sciences 1986, 83(11):3746.

15. Manchado-Rojo M, Weiss J, Egea-Cortines M: Using 23s rDNA to identify contaminations of Escherichia coli in Agrobacterium tumefaciens cultures. Analytical Biochemistry 2008, 372(2):253-254

16. Weiss J, Egea-Cortines M: Transcriptomic analysis of cold response in tomato fruits identifies dehydrin as a marker of cold stress. Journal of Applied Genetics 2009, 50(4):311-319.

17. Mallona I, Lischewski S, Weiss J, Egea-Cortines M: Validation of reference genes for quantitative real-time PCR during leaf and flower development in Petunia hybrida. BMC Plant Biology 2010, 10:4.

18. Delgado-Benarroch L, Weiss J, Egea-Cortines M: The mutants compacta ähnlich, Nitida and Grandiflora define developmental compartments and a compensation mechanism in floral development in Antirrhinum majus. Journal of Plant Research 2009, 122(5):559-569.

19. Mallona I, Egea-Cortines M, Weiss J: Conserved and divergent expression rhythms of CAM related and core clock genes in the Cactus Opuntia ficus-indica. Plant Physiology 2011, 156(4):1978-1989[http://www. plantphysiol.org/content/early/2011/06/15/pp.111.179275.abstract]

20. Hothorn T, Hornik K, van de Wiel M, Zeileis A: coin: Conditional Inference Procedures in a Permutation Test Framework. $R$ package version 0.6-6 2006 [http://CRAN.R-project.org].

21. Wood S: mgcv: GAMs and generalized ridge regression for R. Future 2001, 1:20.

22. Wood S: Stable and efficient multiple smoothing parameter estimation for generalized additive models. Journal of the American Statistical Association 2004, 99(467):673-686.

23. Akaike $\mathrm{H}$ : A new look at the statistical model identification. IEEE Transactions on Automatic Control 1974, 19(6):716-723.

24. Davis J, Goadrich M: The relationship between Precision-Recall and ROC curves. Proceedings of the 23rd International Conference on Machine Learning, ACM 2006, 233-240.

25. Chapman B, Chang J: Biopython: Python tools for computational biology. ACM SIGBIO Newsletter 2000, 20(2):19.

26. Rozen S, Skaletsky H: Primer3 on the WWW for general users and for biologist programmers. Methods in Molecular Biology 2000, 132(3):365-386.

27. Rice $P$, Longden I, Bleasby A, et al: EMBOSS: the European molecular biology open software suite. Trends in Genetics 2000, 16(6):276-277.

28. Chavali S, Mahajan A, Tabassum R, Maiti S, Bharadwaj D: Oligonucleotide properties determination and primer designing: a critical examination of predictions. Bioinformatics 2005, 21(20):3918.

29. Mann T, Humbert R, Dorschner M, Stamatoyannopoulos J, Noble WS: A thermodynamic approach to PCR primer design. Nucleic Acids Research 2009, 37(13):e95[http://nar.oxfordjournals.org/cgi/content/abstract/37/13/ e95].
30. Andreson R, Mols T, Remm M: Predicting failure rate of PCR in large genomes. Nucleic Acids Research 2008, 36(11):e66[http://nar.oxfordjournals. org/cgi/content/abstract/36/11/e66].

31. Pierce K, Sanchez J, Rice J, Wangh L: Linear-After-The-Exponential (LATE)PCR: Primer design criteria for high yields of specific single-stranded DNA and improved real-time detection. Proceedings of the National Academy of Sciences of the United States of America 2005, 102(24):8609.

32. Baskaran N, Kandpal R, Bhargava A, Glynn M, Bale A, Weissman S: Uniform amplification of a mixture of deoxyribonucleic acids with varying GC content. Genome Research 1996, 6(7):633.

33. Varadaraj K, Skinner D: Denaturants or cosolvents improve the specificity of PCR amplification of a G+ C-rich DNA using genetically engineered DNA polymerases. Gene (Amsterdam) 1994, 140:1-5.

34. Benita Y, Oosting R, Lok M, Wise M, Humphery-Smith I: Regionalized GC content of template DNA as a predictor of PCR success. Nucleic Acids Research 2003, 31(16):e99.

35. Tuomi J, Voorbraak F, Jones D, Ruijter J: Bias in the Cq value observed with hydrolysis probe based quantitative PCR can be corrected with the estimated PCR efficiency value. Methods 2010, 50(4):313-322.

36. Ritz C, Spiess A: qpcR: an R package for sigmoidal model selection in quantitative real-time polymerase chain reaction analysis. Bioinformatics 2008, 24(13):1549.

37. Liu W, Saint D: A new quantitative method of real time reverse transcription polymerase chain reaction assay based on simulation of polymerase chain reaction kinetics. Analytical Biochemistry 2002, 302:52-59.

38. R Development Core Team: R: A Language and Environment for Statistical Computing R Foundation for Statistical Computing, Vienna, Austria; 2008 [http://www.R-project.org], [ISBN 3-900051-07-0].

39. Sing $T$, Sander $O$, Beerenwinkel $N$, Lengauer T: ROCR: Visualizing the performance of scoring classifiers 2009 [http://CRAN.R-project.org/ package $=R O C R]$, [R package version 1.0-4].

40. Re AD: compute.es: Compute Effect Sizes 2010 [http://CRAN.R-project.org/ package $=$ compute.es], [R package version 0.2]

41. Program NRA: verification: Forecast verification utilities 2010 [http://CRAN.Rproject.org/package=verification], [R package version 1.31].

doi:10.1186/1471-2105-12-404

Cite this article as: Mallona et al:: pcrEfficiency: a Web tool for PCR amplification efficiency prediction. BMC Bioinformatics 2011 12:404.

\section{Submit your next manuscript to BioMed Central and take full advantage of:}

- Convenient online submission

- Thorough peer review

- No space constraints or color figure charges

- Immediate publication on acceptance

- Inclusion in PubMed, CAS, Scopus and Google Scholar

- Research which is freely available for redistribution

Submit your manuscript at www.biomedcentral.com/submit
C Biomed Central 